\title{
Quel rôle pour les villes petites et moyennes des régions
} périphériques?

Mme Denise Pumain

\section{Citer ce document / Cite this document :}

Pumain Denise. Quel rôle pour les villes petites et moyennes des régions périphériques ?. In: Revue de géographie alpine, tome $87, \mathrm{n}^{\circ} 2,1999$. pp. 167-184;

doi : 10.3406/rga.1999.2950

http://www.persee.fr/doc/rga_0035-1121_1999_num_87_2_2950

Document généré le 06/06/2016 


\title{
Quel rôle pour les villes petites et moyennes des régions périphériques?
}

\author{
Denise Pumain \\ Géographie-cités (CNRS et Université Paris I), 13, rue du Four, F-75006 Paris \\ e-mail : pumain@parisgeo.cnrs.fr
}

Le devenir d'une ville est toujours difficile à prévoir à moyen et long terme. Pourtant, l'étude des transformations de la taille et des activités des villes d'un même territoire fait apparaître des interdépendances considérables dans leur évolution. Ainsi, la situation d'une ville, relativement aux autres villes avec lesquelles elle forme un système, constitue une contrainte forte sur son développement. Evaluée en termes de position géographique, de dimension relative et de spécialisation, cette situation représente d'une part la dépendance historique à l'égard des adaptations antérieures de la ville, de sa trajectoire évolutive, et d'autre part les effets de la concurrence exercée par les autres villes.

Une analyse de l'évolution des villes européennes sur la longue durée illustre ces propositions. Elle montre, par delà l'expansion générale de la taille des villes et de leur nombre, une tendance à la hiérarchisation, c'est-à-dire à l'augmentation des inégalités de la taille des villes. Cette tendance s'explique en partie par les avantages initiaux dans les processus d'adaptation par diffusion des innovations et en partie par les effets de la contraction de l'espace-temps, due à l'augmentation de la vitesse des communications. Cette contrainte spatiale pèse aujourd'hui très lourdement sur la durabilité du développement des villes petites et moyennes. Ainsi, hors catastrophe majeure ou revirement complet des valeurs, certes toujours possibles, tout laisse prévoir que, sans intervention politique forte, le processus séculaire de hiérarchisation des villes, intensifié par l'urbanisation accélérée issue de la révolution industrielle, est appelé à se poursuivre avec la mise en réseaux mondiaux des activités humaines.

Dans ce contexte de la mondialisation, les principales têtes de réseau, les capitales du système monde, consolident leurs positions et s'érigent en mégapoles, métropoles géantes, ou régions mégalopolitaines, aux dépends des villes petites et moyennes qui paraissent condamnées, au mieux à la stagnation, au pire au déclin. Faut-il accepter cette concentration du peuplement, fruit de la concurrence entre les villes, comme le terme d'une évolution inéluctable? Ou bien est-il souhaitable de laisser à chaque ville la possibilité de jouer un rôle spécifique dans le réseau? En dépit du déclin au moins relatif qui menace l'ensemble des plus petites villes européennes, les conditions particulières de la spatialité et les ressources du milieu alpin laissent-elles relativement plus de chances aux villes de cette région (Bätzing/Perlik/Dekleva, 1994)? 


\section{La notion de système de villes}

Concevoir les ensembles de villes comme des systèmes, constitués d'éléments interdépendants, n'est pas une vision nouvelle puisque l'expression bien connue de B. Berry " cities as systems within systems of cities " date du début des années 1960 (Berry, 1964). Déjà au XIX ${ }^{\mathrm{e}}$ siècle des ingénieurs saint-simoniens, comme Jean Reynaud en 1841 , réfléchissaient sur " le système général des villes " (Robic, 1982).

\subsection{DES SYSTÈMFS SPÉCIFIQUES}

La représentation d'un tel système et des interdépendances entre les villes est cependant difficile. Même si pour la commodité nous traçons une limite autour d'un territoire, par exemple un Etat, englobant un réseau urbain de villes différenciées par leurs tailles et leurs fonctions, nous savons certes que les frontières nationales réduisent d'un facteur, qui est évalué à cinq ou six en Europe, les échanges qui sont effectués par delà cette membrane perméable, mais nous savons aussi que les relations de chaque ville avec son environnement varient énormément en portée, selon la taille des villes. Nous avons donc des systèmes assez particuliers où le degré d'ouverture et l'intensité des interactions avec l'extérieur changent selon la taille des éléments.

La notion de système de villes permet d'insister sur les interdépendances qui sont constitutives de la genèse et du fonctionnement des villes. La ville est bâtie sur un site, dont elle utilise certes les ressources locales, mais ce qui fait l'essentiel de sa base économique tient dans l'exploitation des richesses que lui procure sa situation (position dans des réseaux de relations et d'échanges à plus longue distance). En mettant en réseau des sites de ressources complémentaires, la ville impose sa capacité d'accumulation par l'échange inégal, elle réduit les aléas locaux qui sont associés à l'exploitation des ressources de chaque site (Reymond, 1981), et institue par là même, bien avant la lettre, un " développement durable ". En effet, la ville aussi doit innover en permanence, par imitation des autres villes ou par anticipation, afin de maintenir sa position dans le réseau des villes concurrentes. Cette concurrence s'est d'abord manifestée dans l'histoire pour le contrôle politique et économique de territoires, puis pour la captation de marchés qui peuvent aujourd'hui s'organiser, non plus seulement en continuité, dans la région environnant la ville, mais en connexité, dans des réseaux qui associent des sites parfois fort éloignés.

Lorsque des villes appartiennent à un même territoire, c'est-à-dire quand elles participent des mêmes règles générales de fonctionnement politique, économique et culturel, ou encore lorsqu'elles sont étroitement connectées, par delà les frontières nationales, par des relations commerciales, pour le partage des marchés, leur devenir ne peut pas être dissocié de celui des autres villes. La notion de système de villes formalise ces solidarités complexes. 


\subsection{STRUCTURE HIERARCHIQUE ET DIVERSITE DES VILLES}

Ces systèmes présentent des similitudes structurelles très caractéristiques. Les systèmes de villes des différents états du monde sont caractérisés par des propriétés très fortes, relativement invariantes selon les époques ou les territoires considérés. La première est une structure fortement hiérarchisée, qui se traduit par une très grande différenciation de la taille des éléments du système, de quelques milliers d'habitants à plus de 106 dans les grandes métropoles d'aujourd'hui. Le modèle général qui rend compte de cette organisation est une progression géométrique inverse du nombre des villes en fonction de leur taille (ou encore une distribution lognormale de la taille des villes). Les études historiques (De Vries, 1984, Caroll, 1992), voire archéologiques (Fletcher, 1986) ainsi que les comparaisons internationales les plus récentes (Moriconi-Ebrard, 1993), attestent toutes de l'universalité de ce modèle dans l'espace et dans le temps.

Cette structure hiérarchique s'accompagne d'une certaine régularité de l'espacement des unités de peuplement, en général d'autant plus uniforme qu'il s'agit de villes de grande taille. La théorie des lieux centraux (Reynaud, 1841, cité par Robic, 1982, Christaller, 1933) explique cette configuration par le principe de centralité, qui justifie le regroupement en un même lieu de la production des services de même niveau destinés à une population dispersée dans une région complémentaire (ou zone d'influence), dont le centre polarise la clientèle. Les centres sont hiérarchisés du fait de l'existence de plusieurs niveaux de services définis par des portées spatiales (distance que le consommateur accepte de parcourir pour se procurer le service) et des seuils d'apparition (fixés par le volume de clientèle nécessaire à la rentabilité de l'offre de service). Les zones d'influence des centres sont emboîtées, car les centres de niveau supérieur offrent généralement tous les services de portée inférieure. La concurrence entre les centres a pour conséquence une régularité de l'espacement des villes, et la hiérarchie des niveaux de services se traduit par un nombre plus petit et un espacement plus important des centres lorsqu'on s'élève dans la hiérarchie.

Une autre propriété très générale des systèmes de villes, non prise en compte par la théorie des lieux centraux, est la différenciation fonctionnelle, qui à taille égale se traduit par la spécialisation dans des activités différentes des villes appartenant à un même territoire. Cette spécialisation est parfois liée à la prestation des services à la population que prend en compte la théorie des lieux centraux, mais bien plus souvent elle résulte de la concentration d'activités dont la production n'est pas destinée nécessairement à une clientèle voisine. La plupart des activités industrielles sont aujourd'hui dans ce cas, mais aussi certains services comme les transports, ou les services aux entreprises. Si les gisements en bassin (miniers ou touristiques) déterminent parfois des regroupements de villes spécialisées dans le même type d'activités, le plus souvent la localisation des villes spécialisées est très sporadique et ne suit pas de schéma régulier. La spécialisation dans un ou plusieurs types d'activité définit des complémentarités entre les villes d'un même territoire et conditionne en partie leur développement. Les spécialisations majeures apparaissent avec les grands cycles d'innovation technique et économique (par exemple celui de la première révolution industrielle, ou celui du développement de l'électricité), en particulier lorsque qu'ils s'appuient sur des ressources inégalement réparties (comme celles des gisements de charbon ou de minerai, ou encore celles des sites touristiques). 
Le plus souvent, les innovations ont cependant un processus de diffusion qui se conforme à l'organisation hiérarchisée des systèmes de peuplement (Pred, 1977). Même si les innovations peuvent apparaître n'importe où, les grandes villes forment un milieu plus favorable à leur émergence, et possèdent de toute façon une base sociale et économique plus diversifiée qui leur permet d'en bénéficier les premières. Les innovations se banalisent ensuite à l'ensemble du territoire en gagnant progressivement les villes moyennes, puis les petites et parfois les villages. Ce processus de diffusion hiérarchique contribue à maintenir et à amplifier (du fait de l'avantage initial que confere l'adoption précoce d'une innovation) les contrastes hiérarchiques dans la structure des systèmes de peuplement.

\section{Dynamique des systèmes de villes : croissance distribuée et hiérarchisation}

Une théorie évolutive des villes est susceptible de regrouper ces explications classiques de la diversité urbaine en les intégrant dans une perspective de dynamique de systèmes complexes, sous la contrainte de tendances historiques (Pumain, 1997). Les systèmes de villes sont très largement auto-organisés. Leur configuration et leur évolution résultent d'abord des concurrences entre les acteurs qui y ont investi et qui appuient leur action ultérieure sur les ressources mobilisables localement. L'imitation de ce qui a été accompli ailleurs, l'innovation destinée à anticiper sur les concurrents, sont les principaux processus intervenant au niveau "microscopique " de la structure du système, celui des acteurs individuels, et qui se traduisent au niveau " macroscopique " dans des configurations structurelles et des dynamiques semblables, d'un système de villes à un autre, quels que soient les modes de fonctionnement politiques ou économiques des sociétés concernées. Aucune autorité supérieure, aucun principe optimisateur n'exige que le nombre des villes d'un état doive s'ordonner en fonction de leur taille selon une progression géométrique rigoureuse. Pourtant, celle-ci se retrouve, quasiment identique, dans tous les pays du monde (Moriconi-Ebrard, 1993). La structure hiérarchisée de ces systèmes résulte en fait d'un processus évolutif spécifique.

\subsection{LE PROCESSUS DE CROISSANCE DISTRIBUEEE}

L'universalité et la persistance de la structure hiérarchique des systèmes de villes peuvent être expliquées par un processus de répartition de la croissance entre les villes qui résulte directement de la dynamique de concurrence dans laquelle elles sont engagées. Tout se passe comme si, à moyen terme, chaque ville appartenant à un système de villes avait en moyenne la même probabilité de croissance, avec cependant des facteurs de variation multiples, dont la distribution est généralement aléatoire par rapport à la structure du système, et qui sont susceptibles de faire apparaître ici et là, momentanément, de forts noeuds de croissance ou de déclin, absolus ou relatifs. Ce processus, que l'on peut simuler par un modèle stochastique simple (Gibrat, 1931) rend compte à la fois de la forme hiérarchique du système des villes et de l'expansion généralisée des systèmes de villes, sur la 
longue durée. Ceux-ci croissent de façon à peu près similaire dans toutes leurs parties, et s'enrichissent de nouveaux centres, du fait du développement de villages qui franchissent le seuil de l'urbain - plus rarement par la création ex-nihilo de villes nouvelles (ce dernier processus n'est un aspect significatif de la croissance urbaine que dans les pays de colonisation de peuplement). Au total, la croissance se répartit, période après période, dans l'ensemble du système des villes, les fluctuations momentanées se compensent sur le long terme. Cette dynamique rapide très fluctuante explique la persistance des inégalités relatives de dimension des villes, qui restent souvent inchangées pendant plusieurs décennies.

La figure 1 (v.p.172) montre un exemple d'évolution de la trame des villes pour une région européenne grossièrement centrée sur l'arc alpin. Les données de population des villes sont tirées de la base historique mise au point par P. Bairoch et ses collègues (1988), qui retrace l'évolution de la population des villes européennes de plus de 10000 habitants depuis le Moyen-Age jusqu'en 1850, complétées de 1950 à 1990 par celles de la base Géopolis de F. Moriconi-Ebrard (1993). Les cartes illustrent bien la métastabilité de la trame des villes, de 1600 à 1990 , en dépit de l'effet de "remplissage " de l'espace, résultant de la très forte croissance récente des populations urbaines, qui a multiplié le nombre des villes et conduit à augmenter considérablement la surface du cercle représentatif de chacune.

Ce processus de croissance distribuée correspond, qualitativement, à une diffusion généralisée de toutes les transformations socio-économiques qui affectent les activités, la population et la forme des villes. Ainsi, une propriété importante des systèmes de villes est la très grande interdépendance de l'évolution des villes qui appartiennent à un même système. Les changements dans la configuration morphologique (urbanisme), dans les activités (industrialisation ou tertiarisation, passage à la société de l'information), dans la composition sociale (développement du salariat, augmentation des qualifications), dans le nombre des habitants (exode rural, croissance démographique), dans les cultures techniques, voire dans les pratiques des populations citadines, apparaissent très rarement de façon isolée et sporadique dans un seul élément du système. Au contraire, les résultats d'analyses détaillées du changement intervenu dans l'ensemble des villes d'un même territoire montrent une grande homogénéité et simultanéité des transformations qui se sont produites au cours d'une période donnée (Pumain/Saint-Julien, 1978). Comme le changement est presque de même intensité partout, les inégalités et différences qui existent à un moment donné entre les villes se maintiennent sur des durées assez longues. Tout se passe comme si, au cours d'une période donnée, les villes appartenant à un même système subissaient pour l'essentiel une simple translation dans l'espace défini par leurs caractéristiques socio-économiques, le système de villes s'adaptant au changement sans déformer sa structure.

Il peut paraître abusif de parler de système à propos des villes européennes depuis le Moyen-Age, ou même le XVIII ${ }^{e}$ siècle, car on n'avait pas alors de réseau de transport transeuropéen qui aurait unifié l'ensemble. L'application de la notion de réseau urbain en tant qu'ensemble structuré de villes en interaction est d'ailleurs contestée par les historiens et les spécialistes de géographie historique pour toute période antérieure au XVIII siècle, 
Figure1 : Evolution de la population des villes de plus de 10000 habitants.

Sources : Bairoch, Géopolis Conçu et réalisé par Hélène Mathian C.N.R.S - équipe P.A.R.I.S. 
comme étrangère à la réalité des échanges, dont les portées sont alors très réduites, et aux représentations des acteurs (Lepetit, 1988, Martin, 1990). En fait, si les interactions entre les villes ont été longtemps des interactions locales, elles n’en établissaient pas moins de proche en proche des relations de concurrence qui régulaient leur espacement, et des relations réitérées qui expliquent que l'information circule dans tout le système, que les innovations s'y propagent, et que le système se développe sur le long terme en toutes ses parties. La persistance de la trame des villes atteste de l'existence de ces liens de concurrence dynamique entre les villes, qui sont d'abord des liens de concurrence locale, mais susceptibles de produire des régularités à une échelle plus macroscopique, d'où une application possible de la notion de système de villes à des époques bien plus anciennes que celle de la matérialisation des réseaux.

\subsection{UNE CONCENTRATION SECUI.AIRE}

Si la tendance générale à moyen terme est donc à la diffusion de la croissance et des formes qui l'expriment plus qualitativement, une autre tendance apparaît dans l'histoire longue des systèmes de villes. Elle conduit à une accentuation des inégalités entre les villes, à une hiérarchisation accrue du système. Celle-ci apparaît clairement sur la figure 2 (v.p.174), où une ville est cartographiée, non plus comme un cercle qui s'accroît avec le nombre d'habitants, mais par une taille de cercle qui représente l'évolution de la taille de la ville, relativement à celle des autres, indépendamment de la croissance d'ensemble du système. Par ce simple procédé cartographique fixant la taille du cercle représentant la plus grande ville, de population variable selon les époques, et déduisant les cercles représentatifs des autres villes de manière proportionnelle, on souligne l'augmentation du contraste des tailles des villes, déjà perceptible entre 1800 et 1850 et nettement accentué depuis 1950 . Le renforcement des inégalités de taille résulte du processus de diffusion hiérarchique des innovations, mais aussi d'un processus de sélection systématique qui accentue considérablement les effets de la concentration. Ce processus résulte de l'accroissement de la vitesse des communications, qui élargit la portée des relations entre les villes, et par lequel les plus grandes captent, dans leur nouvelle aire d'attraction, la clientèle et les ressources des villes plus petites situées en position intermédiaire entre de plus grands centres urbains. Ainsi court circuitées, les villes petites et moyennes entrent, d'une façon systématique, dans un déclin au moins relatif sinon absolu, de leur population et de leurs activités. Elles perdent de leur importance par rapport aux grandes villes et sont menacées dans le maintien de leur rôle et de leur position dans le système. Certaines d'entre elles seulement se trouvent revitalisées lorsqu'elles entrent dans la périphérie d'une métropole en expansion dont elles deviennent des satellites ou des périphéries dépendantes.

Ainsi, dans un espace-temps, où les possibilités de relation sont exprimées en durées de parcours, le nombre de villes nécessaire à la desserte d'un territoire se réduit au fur et à mesure que la vitesse des transports augmente. Cette réduction n'est que partiellement compensée par les accroissements de la richesse et de la complexité qui, dans le même 
Figure 2 : Evolution de la population des villes de plus de 10000 habitants.

Sources : Bairoch, Géopolis Conçu et réalisé par Hélène Mathian C.N.R.S - équipe P.A.R.I.S

temps, tendraient à faire augmenter la taille et le nombre de villes nécessaires au service des populations et au développement des nouvelles activités (Bretagnolle, 1998). En effet, les deux processus sont d'ampleur inégale : entre le début de la révolution industrielle, l'expansion du produit intérieur brut a permis le remplissage de l'espace par les villes, leurs activités et leur population. Mais l'espace dans lequel se situent ces villes s'est rétracté, dans des proportions bien plus considérables que celles de l'expansion du système du fait de son enrichissement (figure 3, v.p.175). Le processus d'expansion est moins rapide que le 
processus de contraction espace-temps. Certains géographes se sont intéressés depuis longtemps à cette " réorganisation spatiale " liée à la " convergence " de l'espace-temps (Janelle, 1969). L'un des principaux effets de cette gigantesque contraction de l'espace 
dans lequel les villes interagissent est la tendance lourde au déclin relatif des villes petites et moyennes.

\subsection{CONCENTRATION ET MÉTROPOLISATION}

Les manifestations actuelles de la mondialisation sont multiples, qu'il s'agisse de l'internationalisation de la finance, de l'accroissement du commerce international, de l'intensification de l'emprise économique des firmes multinationales, de la multiplication des interactions culturelles, du développement du nombre et de la capacité des moyens de communication à longue distance. Jusqu'à quel point ces processus sont-ils nouveaux pour la dynamique des villes? Certains discours agressifs sur la participation des villes à la mondialisation pourraient laisser croire que celle-ci peut être fortement infléchie par les choix politiques. La volonté des administrations et des acteurs politiques ou économiques locaux de pratiquer l'ouverture internationale, ou encore de favoriser le développement de telle ou telle ville, est en réalité fortement contrainte par les effets de système liés aux interdépendances constitutives du fonctionnement des villes en réseau.

Pourtant, les formes contemporaines des réseaux d'interrelations qui s'établissent entre les villes en soulignent la structure extrêmement hiérarchisée. Par exemple, les implantations des entreprises multinationales dans les villes sont un indicateur de l'intensité des échanges à longue portée qui s'établissent à l'intérieur du système des villes. Or c'est surtout le niveau supérieur de la hiérarchie urbaine qui reste concerné par cette forme de participation aux économies modernes. Ainsi en Europe on trouve très peu d'entreprises filiales d'entreprises multinationales européennes dans des agglomérations de moins de 200000 habitants. Si on considère des expressions moins directement économiques de l'internationalisation des villes, comme par exemple la fréquence des congrès internationaux, en dépit de la présence d'un nombre non négligeable de villes petites et moyennes qui participent à ce mouvement, on observe encore une relative concentration de ce type d'activité à l'intérieur de la hiérarchie urbaine (Rozenblat, 1994).

L'évolution prévisible pour les systèmes de villes contemporains, sous l'effet de la mondialisation, se déduit ainsi des modalités de leur dynamique passée, sous la contrainte d'une nouvelle accélération de la contraction apparente de l'espace physique, lorsque les relations y sont mesurées par le temps de déplacement. Les techniques actuelles permettent d'augmenter considérablement la vitesse des communications inter-urbaines, et ce processus continue d'étendre ses effets à un nombre de villes toujours plus grand. Seule la finitude du monde pourrait être un frein à ce processus. Mais le système des villes du monde est encore loin d'être connecté avec toute la réserve de vitesse disponible. Les conséquences de cette loi " dromologique " sur les systèmes de villes sont un renforcement de leur structure hiérarchique, qui commence par le haut, avec le phénomène identifié depuis la fin des années 1980 comme étant celui de la métropolisation. Ce phénomène n'a en fait rien de nouveau, il n'est que la traduction dans les systèmes des villes de leur adaptation à un nouveau cycle d'innovations, de nombreuses nouveautés techniques, sociales et culturelles accompagnant les progrès de la vitesse des communications et le développement de la société de l'information. Les effets de ce cycle sont appelés à se 
diffuser hiérarchiquement dans le système des villes. La métropolisation contribuera d'autant plus au renforcement des hiérarchies urbaines que la diffusion sera différée dans le temps et sélective spatialement.

Il ne s'agit pas de prétendre qu'aucune transformation n'est à prévoir et que la mondialisation n'aurait pas d'effet sensible sur les positions relatives des villes dans les hiérarchies urbaines. Il ressort par exemple d'une étude comparative de la dynamique des villes de l'Union européenne que, toutes choses égales par ailleurs, les villes se " mondialisent " d'autant plus qu'elles sont grandes, qu'elles ont des fonctions de capitale nationale, qu'elles appartiennent à un pays de dimension modeste, qu'elles ont des fonctions spécialisées dans les activités internationales et qu'elles sont proches d'une frontière (Cattan et al., 1994). La spécialisation fonctionnelle des villes et leur situation géographique sont donc des facteurs importants qui peuvent influencer la répartition entre les villes des effets liés à la mondialisation. Mais les changements que cela implique dans le poids relatif des villes sont très lents et ne retouchent que très partiellement la structure des réseaux urbains.

\section{Le devenir des villes petites et moyennes en Europe}

L'Europe a hérité de sa longue histoire urbaine un système de villes qui comporte un nombre très important de villes petites et moyennes. L'urbanisation de ce continent est caractérisée par un nombre et une densité de villes plus grands qu'ailleurs. Le nombre total des villes européennes de plus de 10000 habitants est trois fois supérieur à celui des villes d'Amérique du nord, pour une population urbaine totale qui n'est que de $40 \%$ supérieure. Leur espacement moyen, d'environ $16 \mathrm{~km}$, est beaucoup plus réduit qu'en Amérique du nord, où il est de l'ordre de $50 \mathrm{~km}$ (Moriconi-Ebrard, 1993). A l'inverse, les plus grandes villes américaines, jusqu'aux trente premières, sont toujours plus peuplées que les villes européennes de même rang. En d'autres termes, les contrastes hiérarchiques entre les tailles des villes sont moins accentués en Europe qu'en Amérique du nord.

Comment interpréter cette considérable différence ? Déjà Christaller (1933) suggérait de la mettre en rapport avec l'histoire du peuplement, et les modalités de déplacement en vigueur au moment où les villes se sont implantées : circulation à pied et à cheval dans le cas des villes européennes, à la vitesse du chemin de fer dans les villes américaines. Dans un continent où le peuplement rural était en outre moins dense, les établissements humains pouvaient d'emblée être plus espacés et, les développements précoces de l'automobile aidant, on explique que les très grandes villes s'y soient plus systématiquement développées.

Quel devenir peut-on imaginer pour les très nombreuses villes petites et moyennes européennes, celles qui ont entre 10000 et 100000 habitants ? En raison de leur longue histoire, ces villes disposent pour la plupart d'un important patrimoine historique, monumental, architectural, et de bonnes conditions d'accessibilité grâce à la haute densité générale des infrastructures, même en pays de montagne. Mais elles sont nombreuses, proches les unes des autres, et donc soumises dans leur développement à forte 
Figure 4 : Variation absolue de la taille des villes (1700-1990)

Sources : Bairoch. Géopolis Conçu et réalisé par Hélène Mathian C.N.R.S - équipe P.A.R.I.S. 
Figure 5 : Variation relative de la taille des villes (1700-1990).

Sources : Bairoch, Géopolis Hélène Mathian C.N.R.S - équipe P.A.R.I.S. 
concurrence. Un diagnostic sur leurs probabilités d'évolution, leurs chances de développement, peut être éclairé par un détour historique, non pas pour retracer la biographie de chacune des ces villes, mais pour en comprendre les dynamiques relatives. Une méthode simple consiste à comparer les trajectoires des villes dans le système.

Sur le long terme, depuis trois siècles, les trajectoires suivies par les villes selon l'évolution de leur population donnent l'impression d'une croissance continue, accélérée à partir de la révolution industrielle qui change significativement le rythme de développement. Sur la figure 4 (v.p.178), les courbes donnent l'impression que la croissance est la même pour les villes quelle que soit leur taille initiale. Mais si on représente l'évolution de la population des villes indépendamment de la croissance d'ensemble du système des villes, c'est-à-dire en retraçant l'évolution de la part que chacune occupe dans la population urbaine totale, ces évolutions relatives sont nettement plus différenciées (figure 5, v.p.179). Dans la région de l'arc alpin, la sélection inter-urbaine s'est effectuée au bénéfice des grandes villes situées à la périphérie de la zone montagneuse. Les trajectoires des villes de cette zone montrent que ce sont surtout les grandes villes multimillionnaires qui sont avantagées, comme Munich, Milan ou Rome (le cas de Vienne mis à part vu ses conditions politiques particulières), même par rapport à des villes de taille encore importante comme Marseille et Lyon. Quant aux villes plus petites, au mieux elles ont maintenu leur position dans le système, mais le plus souvent, elles ont perdu du poids (figure 5, v.p.179).

Ces représentations soulignent qu'il est hors de question d'envisager un renversement d'ensemble de la dynamique des villes petites et moyennes. Il serait trop coûteux, en tout cas pour les sociétés telles qu'elles sont organisées aujourd'hui, d'envisager ce renversement dans l'ensemble de la hiérarchie urbaine. Si les villes petites et moyennes n'ont guère à espérer du fonctionnement ordinaire de la croissance des systèmes urbains, appuyé sur la diffusion des innovations, on est donc contraint d'imaginer que les chances de développement économique qui subsistent pour ce type de villes sont liées au maintien ou au développement d'une spécialisation particulière. Le processus peut avoir des origines diverses, l'implantation pouvant être décidée de l'extérieur, ou résulter d'une initiative interne ou d'un effet de district. On voit alors émerger une adaptation réussie d'un certain type d'activité économique, en général extrêmement " pointue ", souvent articulée sur les marchés mondiaux, dans une petite ville. Un tel processus est très risqué, puisqu'il est soumis au double risque du choix économique initial, toujours incertain, et au risque ultérieur de marquage du lieu, très accentué dans le cas de villes de dimension modeste. Ces effets de marquage sont bien connus dans le cas des spécialisations industrielles, on les imagine moins associés à d'autres spécialisations monofonctionnelles comme celles que créent le tourisme ou certaines activités dites actuellement " de pointe ", mais il n'en existe pas moins.

Ce risque, inhérent à toute spécialisation urbaine, est relativement pernicieux, car il met en jeu d'importants décalages temporels entre la réalité des situations et leur perception. Si l'on représente le processus d'adaptation des villes au flot plus ou moins continu d'innovations qui les contraints d'adopter de nouvelles spécialisations, on peut figurer par un circuit la dynamique qui leur assure une certaine stabilité dans ce processus. Une 
spécialisation monofonctionnelle peut cependant échapper, pour des raisons très diverses, au processus d'adaptation à la nouveauté. La désadaptation n'apparaît pas immédiatement car les représentations urbaines durent en général plus longtemps que les bases économiques qui les ont constituées. Il est donc inévitable que certaines de ces villes spécialisées se retrouvent dans le cercle vicieux d'une autre stabilité qui est celle du déclin, de l'inadaptation. Mais il faut souligner que ce " cycle urbain " n'est jamais complètement équivalent à celui du produit, et que l'émergence d'une restructuration est toujours possible (figure 6).

Il faut donc souligner le rôle des politiques qui anticipent sur ces inévitables cycles du développement-déclin des villes, et qui sachent préserver les capacités du milieu urbain qui les rendent aptes à une réadaptation ultérieure. De là vient l'importance de la notion de développement durable. On sait que l'un des déterminants actuels les plus importants de la durabilité du développement est la situation géographique relative. Dans ce processus, c'est d'abord l'accessibilité qui est importante pour le devenir des villes. S'il y a une politique européenne qui peut enrayer la tendance au renforcement des inégalités hiérarchiques, c'est la diffusion de l'amélioration de la rapidité des moyens de communications dans les territoires. Mais la situation relative des villes dans un réseau local ou régional importe également. On connaît à l'échelle européenne la diversité des trames urbaines régionales, de la disposition relative des villes dans les réseaux locaux et régionaux (Cattan et al., 1994). Cette mesure de la diversité des situations possibles prend aussi du sens à l'échelle des zones rurales (Sanders/Mathian, 1998). Pour le développement des petites villes en milieu rural, deux facteurs paraissent très importants, leur position par rapport aux grandes métropoles d'une part, et leur situation d'accessibilité dans le réseau de communes rurales qu'elles contribuent à animer. 


\section{Conclusion}

La question de la préservation et de l'entretien de leur riche patrimoine de villes petites et moyennes est posée fortement aux Européens, au moment où se dessine avec le Schéma de Développement de l'Espace Communautaire (SDEC) une politique d'aménagement du territoire à cette échelle. Comment préserver un devenir en termes de développement économique pour ces villes ? Face à la puissance de ces tendances " spontanées ", non concertées, non voulues, résultats involontaires d'une multitude de petites actions locales qui produisent à l'échelle des systèmes de villes des effets globaux que nous ne savons pas encore contrôler, la prise de conscience peut jouer un rôle important. En regard de la simplification par le bas des hiérarchies urbaines, de la tendance au déclin des villes petites et moyennes qui ne peuvent plus soutenir la concurrence avec les centres plus grands qui les court-circuitent, leur coupent les ailes en quelque sorte dans leur vie de relations, il n'y aurait guère qu'une volonté politique extrêmement ferme pour contrecarrer des tendances historiques aussi lourdement prononcées.

Dans cette gigantesque rétraction de l'espace de relation dans lequel les villes se placent les unes par rapport aux autres, des occasions sont peut-être offertes par le nouveau cycle d'innovation qui correspond au développement de la société d'information. Une politique d'aménagement volontaire menée dans le souci de préserver les possibilités de développement ultérieur, ainsi que la volonté d'équité en ce qui concerne les conditions de vie offertes aux habitants des métropoles et des villes petites et moyennes, passe aussi par la prise en compte d'indicateurs qui ne sont guère aujourd'hui portés au crédit du développement des territoires. Ces indicateurs qualitatifs, mal évalués, sont relatifs à la qualité de la vie, du cadre de vie, à la disponibilité de temps, ou à l'agrément des relations sociales. En tenir compte permettrait peut-être d'assurer le nécessaire maintien d'une diversité urbaine, dans le monde fini que nous habitons aujourd'hui. 


\section{Bibliographie}

ARCHAEOMEDES, ed., 1998. - Des oppida aux métropoles.

Paris, Anthropos, coll. Villes, 290 p.

BAIROCH P./BATOU J./CHÈVRE P., 1988. - La population des villes européennes de 800 à 1850 . Genève, Droz, Centre d'histoire économique internationale.

BAIROCH P., 1996. - Cinq millénaires de croissance urbaine. Dans : SACHS, I. , ed. : Quelles villes pour quel développement? Paris, PUF.

BÄTZING W./PERLIK M./DEKLEVA M., 1994. — Die Alpen zwischen Verstädterung und Verödung. DISP 34-40.

BERRY B.J.L., 1964. - Cities as systems within systems of cities,

Papers of the Regional Science Association, 13, 147-163.

BRETAGNOLLE A., 1998. - Le système des villes dans l'espace-temps : effet de l'accroissement des vitesses de déplacement sur la taille et l'espacement des villes.

Université de Paris I, thèse de doctorat.

BURA S./GUÉRIN-PACE F./MATHIAN H./PUMAIN D./SANDERS L., 1996. Multi-agent systems and the dynamics of settlement systems.

Geographical Analysis, 2, 161-178.

CARROLL G.R., 1982. - National City-size distributions : what do we know after 67 years of research? Progress in Human Geography, 6, 1.

CATTAN N./PUMAIN D./ROZENBLAT C./SAINT-JULIEN T., 1994. -

Le système des villes européennes. Paris, Anthropos, coll.Villes, 201 p.

CHRISTALLER W., 1933. - Die zentralen Orte in Süddeutschland. Iena, Fischer.

DE VRIES J., 1984. - European Urbanization, 1500-1800.

London and Cambridge (Mass.).

FLETCHER R., 1986. - Settlement in Archaeology : world-wide comparison.

World Archaeology, 18, 1, 59-83.

GIBRAT R., 1931. — Les inégalités économiques. Paris, Sirey.

JANELLE D.G., 1969. - Spatial reorganization : a model and concept.

Annals of the Association of American Geographers, 348-368.

JUILLARD E., 1970. — L'armature urbaine de la France préindustrielle.

Bulletin de la Faculté des Lettres de Strasbourg, 6.

MORICONI-EBRARD F, 1993. - L'urbanisation du monde. Paris, Anthropos, coll. Villes.

MORICONI-EBRARD F., 1994. - Geopolis, pour comparer les villes du monde.

Paris, Anthropos, coll. Villes. 
PRED A., 1977. - City systems in advanced societies. London, Hutchison.

PRUDHOMME R., 1996. — Le PIB des grandes villes du monde.

Dans : PUMAIN D./GODARD F., eds., Données urbaines 1, 73-76.

PUMAIN D., 1974. - Histoire de la géographie québecoise.

Université de Paris I, thèse de 3e cycle.

PUMAIN, D./SAINT-JULIEN T., 1978. — Les dimensions du changement urbain. Paris, CNRS, 202 p.

PUMAIN D./MORICONI-EBRARD F., 1997. - City size distributions and metropolisation. Geojournal, 43, 4, 307-314.

PUMAIN D., 1997. — Vers une théorie évolutive des villes.

L'Espace Géographique, 2, 119-134.

PUMAIN D., 1998. — La géographie saurait-elle inventer le futur?

Revue européenne des Sciences sociales, 110, 53-70.

RECLUS E., 1895. - The evolution of cities. The Contemporary Review, 67, 2, 246-264.

REYMOND H., 1981. - Une problématique théorique, in Isnard H. Racine J.B. Reymond H. Problématiques de la géographie, Paris, PUF.

REYNAUD J., 1841. - Villes, in Encyclopédie nouvelle, Paris, Gosselin, t.VIII, 670-687 (cité par Robic, 1982).

ROBIC M.-C., 1982. - Cent ans avant Christaller, une théorie des lieux centraux. L'Espace Géographique, 1, 5-12.

ROZENBLAT C., 1994. - The formation of a European urban system, in G. Braun (ed), Managing and Marketing of Urban Development and Urban Life, Berlin, Dietrich Reimer Verlag, 137-156.

SANDERS L./MATHIAN H., 1998. - Attraction urbaine et contexte d'urbanisation, in Pumain D. Mattéi M.-F., eds., Données urbaines, 2, Paris, Anthropos-Economica, 397-410.

SASSEN S., 1991. - The Global City. Princeton, Princeton University Press.

SUAREZ-VILLA L., 1988. - Metropolitan Evolution, Sectoral Economic Change, and the City-Size Distribution. Urban Studies, 25, 1, 1-20. 